\title{
Modélisation synthétique quantitative de
} plusieurs années d'activité. Quelle articulation entre signification en situation et complexité longitudinale?

Quantitative synthetic modelling of several years of activity. What relationship between meaning in situation and longitudinal complexity?

\section{Julien Guibourdenche}

\section{OpenEdition}

\section{Journals}

Édition électronique

URL : https://journals.openedition.org/activites/5522

DOI : 10.4000/activites.5522

ISSN : $1765-2723$

\section{Éditeur}

ARPACT - Association Recherches et Pratiques sur les ACTivités

\section{Référence électronique}

Julien Guibourdenche, « Modélisation synthétique quantitative de plusieurs années d'activité.

Quelle articulation entre signification en situation et complexité longitudinale? », Activités [En ligne],

17-2 | 2020, mis en ligne le 15 octobre 2020, consulté le 25 août 2021. URL : http://

journals.openedition.org/activites/5522 ; DOI : https://doi.org/10.4000/activites.5522

Ce document a été généré automatiquement le 25 août 2021.

\section{(i)@()}

Activités est mis à disposition selon les termes de la licence Creative Commons Attribution - Pas d'Utilisation Commerciale - Pas de Modification 4.0 International. 


\section{Modélisation synthétique} quantitative de plusieurs années d'activité. Quelle articulation entre signification en situation et complexité longitudinale?

Quantitative synthetic modelling of several years of activity. What relationship between meaning in situation and longitudinal complexity?

Julien Guibourdenche

\section{NOTE DE L'ÉDITEUR}

Article soumis le 2 mars 2020, accepté le 23 mai 2020

\section{NOTE DE L'AUTEUR}

L'auteur est seul responsable des propos tenus dans cet article et déclare n'avoir aucun conflit d'intérêt.

L'auteur tient à remercier les participants à cette étude ainsi que tous les acteurs du projet Smart Electric Lyon, plus particulièrement Pascal Salembier, Germain Poizat, Mariane Galbat et Yvon Haradji. Le projet Smart Electric Lyon a été soutenu par l'ADEME (Agence française de l'environnement et de la maîtrise de l'énergie) dans le cadre des Investissements d'Avenir. 


\section{Introduction}

1 Malgré l'intérêt de l'ergonomie pour les dynamiques temporelles, de nombreux enjeux requièrent encore une meilleure compréhension des temps longs et temporalités multiples de l'activité humaine : la santé et les maladies professionnelles (Mardon, Buchmann, \& Vokoff, 2013), la gestion temporelle (Zara-Meylan, 2013, 2016), le développement de pratiques professionnelles (Chizallet, Prost, \& Barcellini, 2019), ou bien encore la soutenabilité (Fréjus, 2019; Thatcher, Guibourdenche, \& Cahour, 2019). Le problème général tient à ce que comprendre l'activité signifie la situer dans l'« ici et maintenant » de son émergence tout en tenant compte du fait que cette dernière est «structurée par et structurante de » temporalités lointaines et plurielles. Les travaux sur les cours de vie d'agriculteurs (Barbier, Cerf, \& Lusson, 2015) ou la diachronie d'activités narratives (Bationo-Tillon, Folcher, \& Rabardel, 2010) l'exemplifient. Daniellou (2005) parle aussi de l'activité comme d'une tranche de vie (a slice of life). Cet aspect est récurrent dans les études longitudinales de l'activité et fait naitre d'importants enjeux pour la recherche en ergonomie. Outre les enjeux conceptuels, il est souvent impossible d'analyser finement toutes les données (verbatims, vidéos, photos, statistiques, etc.) sur plusieurs années. On peut alors passer de niveaux analytiques locaux à généraux sans réelle théorie explicite de la complexité longitudinale de l'activité étudiée. Cela n'enlève rien à l'intérêt des études actuelles, mais une théorie générale des dynamiques longitudinales de l'activité permettrait peut-être d'améliorer ou faciliter celles à venir. À l'inverse, il est parfois tentant d'appliquer des cadres prédéfinis (e.g., Harbich \& Hassenzahl, 2017) sur de vastes étendues temporelles pour les couvrir de façon plus complète. Mais l'interprétation des données en regard de l'activité réellement construite par les acteurs pose alors des questions épineuses. Comment s'assurer de leur validité du point de vue de l'acteur en situation de travail?

2 Ces enjeux relancent ici l'intérêt de la réflexion de Theureau (2006) sur l'articulation entre modèle analytique et modèle synthétique dans le programme de recherche cours d'action. En clôture de Cours d'action - Méthode développée, Theureau (2006) introduit plusieurs catégories distinctes de méthodes et de modèles (analytique ou synthétique, empirique ou pratique, etc.). Cela fait suite à ses réflexions sur le concept de modèle (Badiou, 1969), sur les modèles d'analyse des situations de travail complexes (cf. Amalberti, de Montmollin, \& Theureau, 1991), ainsi que sur les modèles et la complexité dans une perspective énactive (Theureau, 2005).

Un modèle empirique analytique concrétise les catégories descriptives du terrain d'étude et contribue ainsi à révéler des caractéristiques spécifiques de l'activité (Theureau, 2006). Ce type de modèle est extrêmement répandu en ergonomie comme en analyse d'activité en général. L'objet théorique cours de vie a donné lieu à différents modèles empiriques d'évolution de l'activité sur le temps long (e.g., Barbier et al., 2015 ; Haué, 2003). Le plus souvent issu d'une méthode d'analyse qualitative, un modèle empirique analytique est descriptif et parfois graphique. Il ouvre aussi sur des propositions pratiques de transformation pour un terrain d'étude/intervention particulier. Mais sa construction est limitée par le terrain et par le temps d'analyse qualitative des données, d'autant plus lorsqu'il s'agit de travailler sur des temporalités longues de plusieurs années impliquant une profusion des données. 
4 Un modèle empirique synthétique est moins précis sur le contenu de l'activité d'un acteur particulier, car il est issu d'une application systématique de catégories génériques préexistantes à un ensemble de données (les catégories dites " de codage " pour reprendre un terme répandu). L'archétype du modèle synthétique est mathématique : constitué d'un faisceau d'hypothèses supposé complet relativement au domaine d'étude, cohérent et ouvrant sur des raisonnements déductifs (Theureau, 2006). Il s'accompagne le plus souvent de «montages matériels» (graphes, diagrammes, etc.) qui le représentent ou l'imitent. Plus "générique», le modèle synthétique permettrait de rendre compte de façon plus complète et homogène de la production d'un phénomène sur un espace-temps étendu comme des années. En revanche, s'il est utilisé seul, il fait courir le risque d'une interprétation fallacieuse du contenu spécifique de l'activité et de l'expérience vécue par l'acteur dans cette activité (Theureau, 2006) : ses préoccupations, son ressenti, son interprétation de la situation, ses projections et attentes...

$5 \quad$ L'idéal serait alors d'articuler ces deux types de modèles de manière à développer des connaissances ancrées dans les situations des acteurs (analyse qualitative) aussi bien que dans les dynamiques de long terme (ouvertes à caractérisation synthétique mathématique). Les catégories des modèles analytiques peuvent et doivent servir à construire « des modèles synthétiques plus pertinents que ceux qui seraient construits au moyen de la seule intuition du modélisateur» (Theureau, 2006, p. 344). Mais comment procéder et que peut-on attendre concrètement de cette articulation? Dans le cadre d'une recherche longitudinale sur l'appropriation, cet article discute des conditions de production et de l'utilité d'une telle articulation. Après avoir positionné notre travail entre les approches de l'activité et celles de la complexité temporelle en ergonomie (section 2), l'article expose en section 3 notre cadre de modélisation analytique-synthétique développée initialement dans le projet Smart Electric Lyon. La section 4 montre que le passage à une modélisation synthétique quantitative valide se joue tout d'abord dans les travaux de recueil, de traitement des données et de cadrage des analyses des résultats. Les résultats de la section 5 démontrent que l'articulation proposée permet de synthétiser plusieurs années d'activité en restant capables de spécifier la signification des situations locales qui conduisent à des dynamiques d'adoption ou de rejet des systèmes, ainsi que de fournir de nouvelles hypothèses alimentant une démarche déductive. Sur ces bases, la discussion de la section 6 ouvre des perspectives pour poursuivre le développement des approches de la complexité temporelle entre une phénoménologie de l'activité et/ou de l'expérience vécue et sciences de la complexité.

\section{Quels modèles de complexité longitudinale pour l'activité individuelle?}

6 Les enjeux de modélisation longitudinale de l'activité engagent différents positionnements conceptuels et méthodologiques en ergonomie et human factors relativement au temps et à l'activité humaine. Tout en nous intéressant aux modèles des sciences de la complexité et des human factors (mathématiques, synthétiques, abstraits, chronologiques), notre articulation analytique-synthétique s'inscrit d'abord dans une tradition d'analyse d'activité. 


\subsection{La complexité longitudinale : quel lien aux sciences de la complexité ?}

7 Les sciences de la complexité et leurs modèles synthétiques peuvent-ils fournir une partie des outils pertinents pour comprendre différemment les dynamiques longitudinales de l'activité ? En sciences des systèmes complexes, Boeing (2016) laisse supposer que l'approche visuelle des modèles mathématiques de systèmes dynamiques non-linéaires permettrait de détecter des structures « cachées » dans les ensembles de données temporelles complexes. Mais peut-on assimiler l'activité individuelle à un système dynamique non-linéaire par exemple? Dans la perspective des human factors, Guastello (2017) soutient que la théorie des systèmes dynamiques non-linéaires permettrait de reformuler les problèmes d'ergonomie qui ont trait à l'évolution temporelle des systèmes complexes : les accidents du travail, la résilience d'un système, la charge cognitive, les degrés de liberté dans le mouvement, ou encore la coordination d'une équipe. Ce dernier auteur et Walker, Stanton, Salmon, Jenkins et Rafferty, (2010), en appellent à une orientation encore plus assumée de l'ergonomie vers les sciences de la complexité, afin d'envisager autrement les problèmes qui lui sont posés. Thatcher (2016) semble également aller en ce sens, en appelant à mobiliser les modèles de la Network Analysis (une forme d'analyse des systèmes complexes) pour appréhender les dynamiques de transformation temporelle de système-de-systèmes durables. Ces appels s'inscrivent dans un mouvement plus large d'évolution en direction des sciences de la complexité, incluant les sciences de l'éducation (e.g., Hilpert \& Marchand, 2018) ou la psychologie sociale dynamique (e.g., Wiese, Vallacher, \& Strawinska, 2010) par exemple.

La connaissance de la dynamique longitudinale de l'activité humaine au sens d'une construction située, cultivée et historicisée de pratique, technique et signification en action, ne fait pas partie des domaines d'application cités ou prisés par ces auteurs. Ce n'est simplement pas l'approche retenue. Par conséquent, avant d'appliquer tel ou tel modèle mathématique de complexité pour synthétiser une dynamique longitudinale de l'activité, il convient de s'interroger sur l'articulation analytique-synthétique: ses fondements conceptuels, méthodologiques, etc.

9 Hilpert et Marchand (2018) proposent une catégorisation intéressante des modèles ou «montages matériels» (Theureau, 2006) possibles concernant les systèmes complexes. Les graphes de séries temporelles semblent pertinents pour la description temporelle du comportement d'un élément particulier d'un système complexe (e.g. un individu dans un système de production). Les graphes de l'analyse de réseaux et de la modélisation dynamique intéressent des niveaux d'organisation différents dépassant notre étude. La suite de cet article initie la réflexion en articulant des catégories analytiques d'activité à une synthèse quantitative passant par une série temporelle. Que permet cette articulation? Que cache-t-elle et qu'implique-t-elle vis-à-vis de l'évolution de l'activité humaine entre situation et temps long?

\subsection{Complexité longitudinale en ergonomie francophone centrée sur l'activité}

10 L'ergonomie de langue française est riche d'approches très diverses des dynamiques longitudinales de l'activité et des systèmes complexes. Il faut noter une évolution 
générale du statut des modèles, en arrière-plan de cet article. Modéliser signifie bien encore réduire, décrire, expliquer ou simuler (Amalberti et al., 1991). Mais les dernières décennies ont aussi souligné l'importance de la modélisation en tant qu'action dans des contextes d'intervention de plus en plus participatifs et réflexifs : pour la médiation des connaissances (à soi-même, à autrui), pour dépasser la contingence des connaissances de la situation et pour co-construire des perspectives sur des bases empiriques fermes, mais «mises à l'épreuve» (Albaladejo et al. , 2009). En matière de «modèles longitudinaux » de l'activité, on trouve alors un panel très riche, allant de descriptions très locales par l'analyse de verbatims à des simulations multi-agent de l'évolution chronologique de tâches et d'effets de l'activité. Nous décrivons ci-dessous quelques approches et modèles les plus pertinents pour cet article.

11 Les verbalisations et les verbatims des acteurs peuvent permettre de caractériser la complexité longitudinale de l'activité. Zara-Meylan (2016) caractérise ainsi la complexité des temporalités longues et plurielles structurant l'activité d'horticulteurs en mobilisant une approche radicalement qualitative et phénoménologique. Par l'analyse abductive progressive de verbatims, elle dévoile des enchevêtrements temporels de cadres et les configurations complexes de l'activité des horticulteurs. Cela lui permet de mettre en avant plusieurs types de temporalités en jeu dans cette activité au long cours: temps poussé par le passé, tiré par l'avenir, temps d'ouverture de possibles... Ces temporalités nous semblent faire écho à celles d'actualité, potentialité et virtualité dans l'approche sémiologique du cours d'action (Theureau, 1992) inspirée de Peirce, ou à celles de virtuel, réel et concevable dans les travaux de Chizallet et al. (2019). Ces notions sont susceptibles, parmi d'autres, d'une caractérisation par verbatims.

12 Les chroniques temporelles, extrêmement répandues en ergonomie, ne relèvent pas d'une évidence bien qu'elles soient utiles à la création d'un espace de référence commun sur les dynamiques longitudinales. À la différence des verbalisations, elles impliquent, pour tout ou partie, une représentation linéaire homogène qui n'aborde pas le vécu du temps figuré (Zara-Meylan, 2016). Leur principe même est d'inclure le temps chronologique dans la modélisation, ce qui implique une réflexion de fond sur le statut du «Chronos » vis-à-vis du temps vécu et du temps de l'activité : du «Kairos ». Cependant, il serait réducteur de limiter le temps représenté par les « chroniques » au seul Chronos et notre étude cherche à articuler ces deux formes de temps. La mise en chroniques peut intégrer de façon forte des catégories d'expérience vécue et des contenus d'activité (e.g., Barbier et al., 2015; Chizallet et al., 2019; Haué, 2003). Par ailleurs, leurs atouts synthétiques sont non-négligeables pour représenter des dynamiques de plusieurs années dans un espace restreint (e.g., moins d'une page).

Enfin des articulations d'approches modélisatrices de l'activité humaine et de la complexité des systèmes (Amalberti et al., 1991; Theureau, 2005) fournissent d'autres perspectives pour la recherche empirique et technologique en ergonomie. Dans cette veine, Haradji, Guibourdenche, Reynaud, Poizat, Sabouret, Sempé et al. (2018) articulent modèles analytiques situés de l'activité humaine et modélisations mathématiques numériques à base de système multi-agent dans une perspective technologique de simulation de consommations d'électricité à large échelle. En ergonomie cognitive, des situations sportives Bourbousson (2013) ont proposé des pistes permettant de travailler sur l'activité collective de façon articulée entre anthropologie cognitive et théories des systèmes dynamiques. Elles montrent que les concepts, méthodes et modèles de la 
complexité peuvent porter des conceptions fécondes dès lors qu'elles sont appuyées par des regards où l'activité humaine est prise en compte de façon située. Quoi qu'il en soit, il manque, à notre connaissance, des modèles mathématiques synthétiques de la dynamique longitudinale de l'activité individuelle : un faisceau d'hypothèse permettant d'entrer dans une démarche déductive à propos du «devenir» d'une activité individuelle sur des temps longs de plusieurs années. Si l'activité est dans un état A à l'instant $t$, à quoi doit-on s'attendre à l'instant $t+n$ et pourquoi ?

Dans ce contexte, si la construction de modèles adéquats de la complexité est un enjeu fort de l'intervention et de la recherche en ergonomie (Amalberti et al., 1991; Wisner, 1972), qu'en est-il pour la dynamique longitudinale de l'activité individuelle-sociale? Est-il possible d'articuler un modèle de la signification de l'action à une approche plus mathématique de son évolution? À quelles conditions et pour quels apports?

\section{Un observatoire de la dynamique longitudinale de qualité d'expérience vécue}

Cet article s'appuie sur un "pas de côté » réalisé lors du projet Smart Electric Lyon (Guibourdenche, Poret, Poizat, Motté, Haradji, Salembier et al., 2019) en s'inspirant d'apports issus du programme de recherche empirique et technologique Cours d'Action (Theureau, 2005, 2006) et d'autres travaux en psychologie ergonomique (Cahour, Salembier, \& Zouinar, 2016) ainsi qu'en long-term user experience (Harbich \& Hassenzahl, 2017 ; Karapanos, Martens, \& Hassenzahl, 2012 ; Kujala, Roto, Väänänen-Vainio-Mattila, Karapanos, \& Sinnelä, 2011).

\subsection{Une étude de l'appropriation des systèmes de gestion d'énergie dans le résidentiel}

La question de complexité longitudinale nous a été posée à travers celle de l'appropriation des systèmes de gestion d'énergie résidentielle. L'appropriation est approchée ici de façon double : comme processus d'intégration au monde et à la culture propres d'un acteur (Theureau, 2006) et comme donnant lieu à adoption/rejet d'une technique. Par exemple, comment un acteur apprend-il à déclencher un mode de gestion économique de son habitat? Comment intègre-t-il le mode de fonctionnement du système à ses propres connaissances et modalités d'appréhension du monde? En quoi cette intégration progressive ouvre-t-elle sur une adoption ou un rejet du système?

17 L'appropriation des systèmes de gestion d'énergie fournit une situation d'étude intéressante de la complexité des dynamiques temporelles de l'activité humaine. Par exemple, elle tient pour partie à ce que le système de gestion rende l'acteur capable de mieux déterminer et maîtriser ses consommations à travers le temps. Mais déterminer l'impact sur ses consommations d'énergie nécessite souvent de disposer de données couvrant deux saisons de chauffe (hivers) ou de consommation (années); afin de pouvoir comparer deux mois similaires d'années différentes, ou deux années différentes par exemple. Que se passe-t-il durant ces 2 ans pour l'acteur? À partir de quelles attentes de départ? De nouvelles attentes naissent-elles après les premières 
utilisations? À quoi ressemble le processus d'appropriation relativement à ces attentes? Qu'est-ce qui explique l'adoption et le rejet au bout de plusieurs années ?

Par ailleurs, les moments d'appropriation ${ }^{1}$ caractérisés dans notre étude engagent une socialité et une technicité beaucoup plus vaste à travers le temps que les seuls moments d'utilisation du système technique. Ils peuvent prendre la forme de contacts et communications de l'acteur (participant) avec le fournisseur d'énergie, avec le constructeur du système, avec l'installateur ou le réparateur, voire avec ses collègues, ses voisins ou amis. Comment tous ces moments interagissent-ils les uns avec les autres à travers le temps? Revêtent-ils la même importance du point de vue de l'activité d'appropriation de l'acteur? Quelles sont les zones de confiance et d'incertitudes dans l'évolution temporelle d'une telle trajectoire d'appropriation? Nous revenons ici à des questions en partie communes avec les approches longitudinales en ergonomie, évoquées en introduction.

Pour travailler ces questions, nous nous appuyons sur une articulation analytiquesynthétique touchant les différents plans d'une recherche en ergonomie : conceptuels, méthodologiques (recueil et traitement), empiriques et technologiques.

\subsection{Un ancrage dans l'analyse qualitative de l'expérience vécue de l'activité}

Sur les plans conceptuels, nous nous appuyons sur la notion d'expérience vécue de l'activité, qui a globalement structuré notre observatoire longitudinal, puis sur l'hypothèse d'une "dynamique de qualité d'expérience vécue ». L'expérience vécue d'une activité est définie comme le "flot d'actions, d'émotions, de pensées et de sensations perceptuelles générées pendant la réalisation d'une activité, dont le sujet est ou peut être conscient " (Cahour et al., 2016). L'expérience vécue de l'activité dans les moments d'appropriation des systèmes de gestion d'énergie permet de rendre compte des besoins, contraintes et attentes des acteurs participants à l'étude, en respectant le principe du primat de l'intrinsèque (Theureau, 2005, 2006).

21 Entre 2014 et 2015, une période analytique d'un an et demi (Figure 1) s'est ouverte durant laquelle les principes suivants ont été mis en œuvre : recueils en situation par observation, verbalisations simultanées et post-hoc, entretiens de remise en situation, analyse qualitative des données. Cette période nous a menés (Figure 2, point $\mathrm{A}$ ) à la réalisation de diagnostics analytiques sur les situations, besoins, contraintes et attentes des participants, ainsi que vers des pistes de conception pour le service et les systèmes techniques. 
Figure 1 : Observatoire longitudinal associant des méthodes classiques pour l'analyse qualitative d'une expérience vécue (observations in situ, traces d'activité notées dans un carnet, confrontation aux traces, entretiens, remise en situation) (icônes noirs) et quantitatifs-qualitatifs synthétiques (icônes blancs) permettant de documenter ce que nous avons appelé ensuite la qualité de l'expérience vécue. 43 sessions de recueil ont été effectuées avec 13 participants de 2013 à 2016. Figure 1: Longitudinal observatory combining classic methods for the qualitative analysis of a lived experience (in situ observations, traces of activity noted in a notebook, confrontation with traces, interviews, role-playing) (black icons) and synthetic quantitative-qualitative methods (white icons) making it possible to document what we then called the quality of the lived experience. 43 collection sessions were carried out with 13 participants from 2013 to 2016

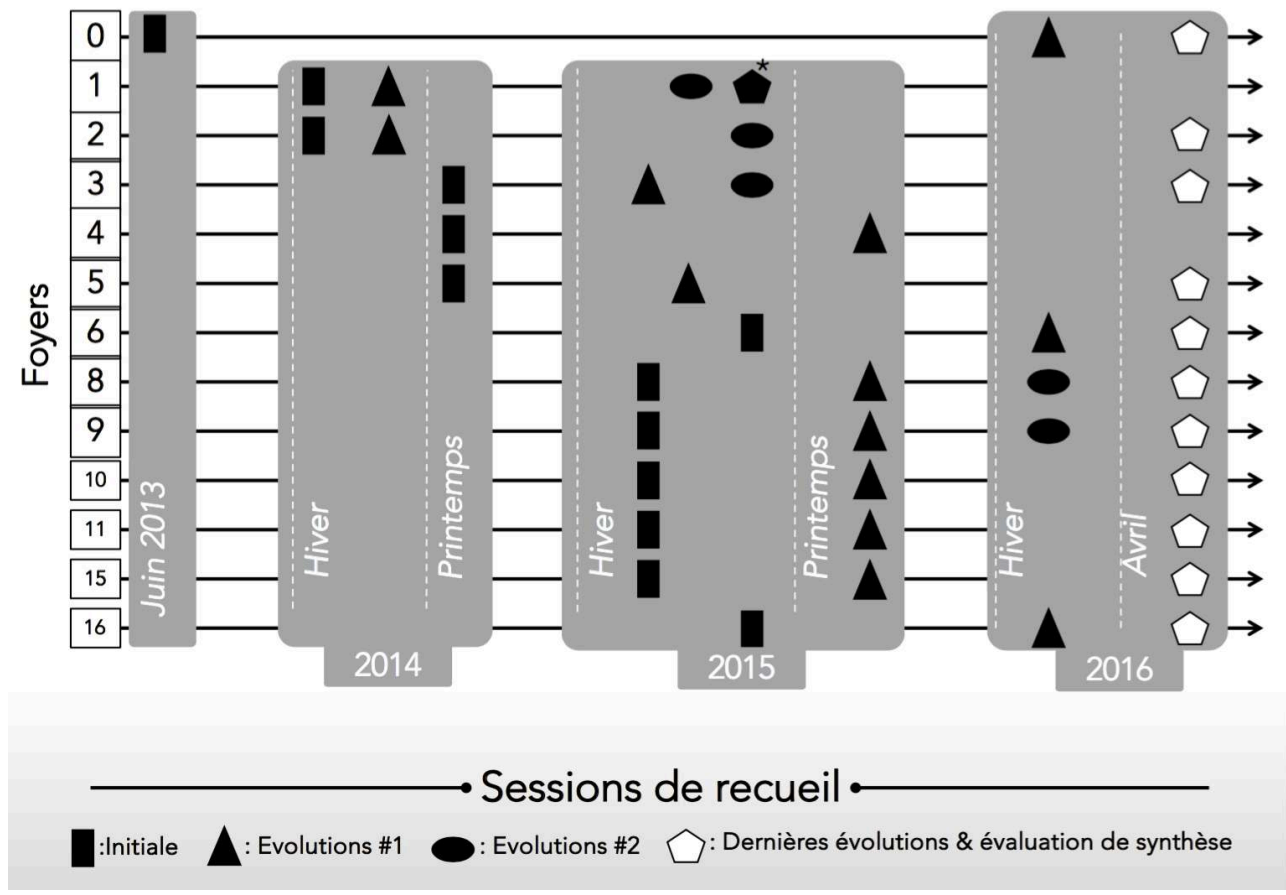

Cette période analytique a aussi vu naitre les premières formalisations graphiques des dynamiques d'appropriation (non présentées ici), caractérisant les interactions entre les attentes et préoccupations des participants avec les évènements significatifs dans les moments d'appropriation au fil du temps: une panne, une baisse de température, un départ en vacances... Arrivés à la fin de nos analyses de 2014 et 2015, les premiers diagnostics sont posés sur l'appropriation. Nous souhaitons alors développer la recherche méthodologique sur ces aspects longitudinaux. Pour repère, le corpus de données transcrites complètement (verbatims, photos, observations) contient environ 600 pages à l'été 2015. Nous introduisons alors une première représentation synthétique dimensionnelle sous forme de radar dans la discussion du rapport d'étape du projet. Nous indiquons que des dimensions caractérisant la qualité de l'expérience (facilité, confort thermique) nous paraissent revenir de façon récurrente dans les moments d'appropriation pour orienter positivement ou négativement les trajectoires vers du rejet ou de l'adoption. Il s'agit du premier tournant entre la fin de l'analyse qualitative et le début de l'approche synthétique de la dynamique de qualité d'expérience vécue (Figure 1 pentagones blancs et Figure 2 points $B, C$ et $D$ ). 


\subsection{La qualité de l'expérience vécue : un objet d'étude ouvrant vers une synthèse}

La notion de "qualité de l'expérience vécue » nous permet ici un pas de côté, car il aurait été impossible de systématiser une analyse de cours de vie sur 13 foyers pendant 3 ans. Le choix fut de réaliser un travail d'analyse systématique appuyant ensuite l'établissement d'une synthèse, selon le mouvement proposé par Theureau (2006, p. 347 , cf. section 1). La notion de « qualité d'expérience vécue » n'était qu'implicite au départ de ce travail. Elle se rapproche de ce qu'on peut entendre plus usuellement par "expérience utilisateur». Par "qualité d'expérience vécue», nous entendons un ensemble de dimensions qualitatives thématisant l'expérience vécue, mais ne lui donnant pas forme intrinsèque, cette forme étant réservée à l'expérience vécue ellemême, la conscience préréflexive et l'émergence des significations dans l'action [informo pour Theureau (2006)]. Les approches en UX research, psychologie ergonomique ou ergonomie fournissent des dimensions concernant les émotions, le plaisir, l'hédonisme, de façon complémentaire aux «classiques» utilité et facilité d'utilisation.

Figure 2 : Évolution de notre méthode d'étude longitudinale de l'activité de l'analyse vers la synthèse.

Figure 2: Evolution of our longitudinal study method from analysis to synthesis

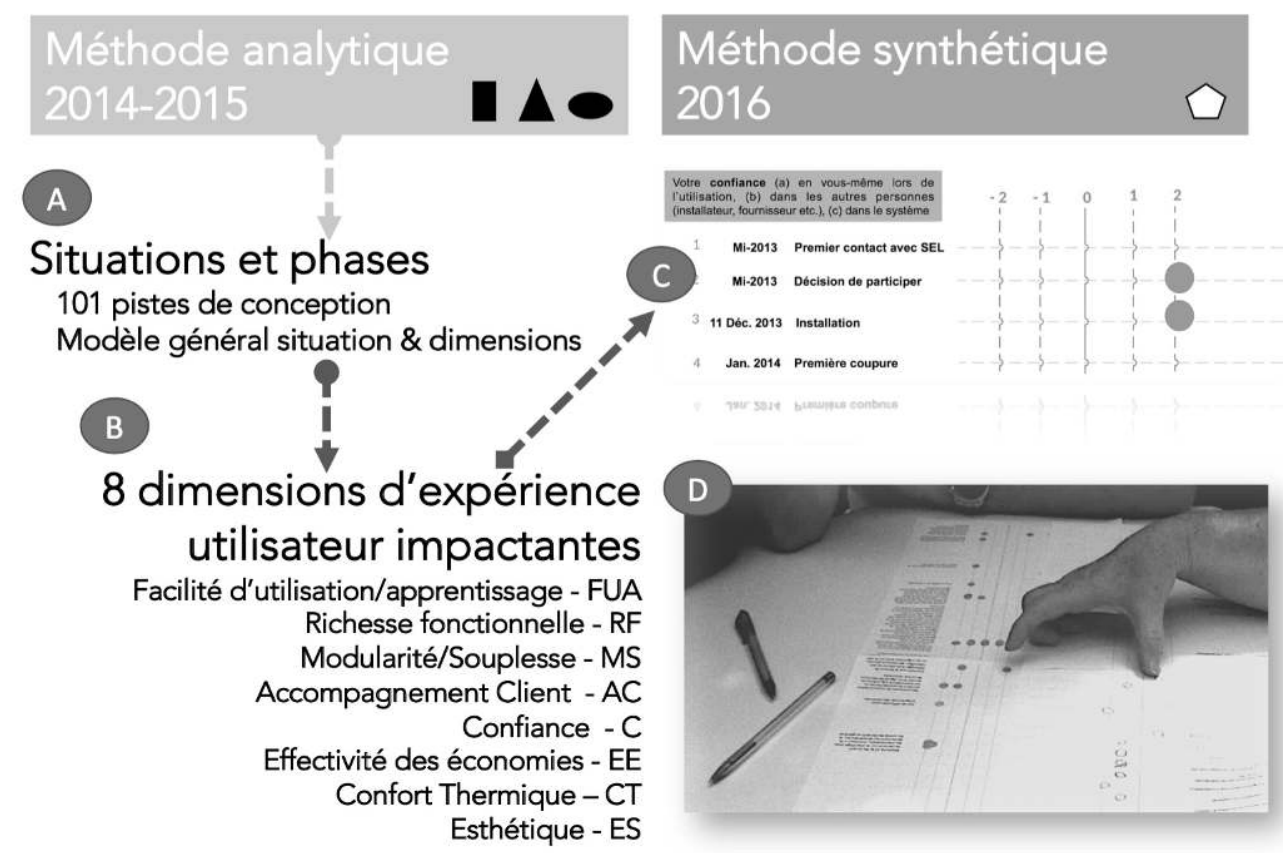

Nous n'avons pas repris ces dimensions directement, car la qualité de l'expérience vécue est susceptible d'une caractérisation par analyse et synthèse "inventives " ${ }^{2}$ (Theureau, 2005). Notre synthèse s'appuie sur 8 dimensions issues du terrain (extraites de notre corpus de 600 pages, Figure 2 point $B$ ), sur un développement méthodologique (points C et D) librement inspiré des UX curves et de iScale (Karapanos et al., 2012; Kujala et al., 2011), et sur les principes des entretiens de confrontation aux traces de l'activité (Cahour et al., 2016; Theureau, 2010). Sur la base du corpus des 28 recueils effectués entre 2013 et 2015, ce développement a été réalisé collectivement et de façon contradictoire dans l'équipe. 
25 Figure 2, Point A - Comme expliqué en section précédente, les années 2014 et 2015 ont été dédiées à la réalisation de diagnostics analytiques sur les situations, besoins, contraintes et attentes des participants, ainsi qu'à la formalisation des pistes de conception pour le service et les systèmes techniques. À l'été 2015, le travail de recherche sur la caractérisation de la qualité de l'expérience débute par un nouveau traitement du corpus de 600 pages constitué jusque-là.

Figure 2, Point B - En février 2016, 8 dimensions ont ainsi été identifiées de façon consensuelle dans l'équipe : la facilité d'utilisation et d'apprentissage (FUA), l'effectivité des économies (EE), le confort thermique (CT), l'accompagnement (AC), la confiance (C), la modularité et la souplesse (MS), la richesse fonctionnelle d'ensemble (RF) et l'esthétique (ES).

27 Figure 2, point $\mathbf{C}$ - Afin de donner corps à la dynamique de « qualité de l'expérience vécue » à travers le temps, nous avons développé une chronique nommée Chronovec (Figure 2, points $C$ et D). Pour chaque verbalisation, nous avons :

a. identifié et nommé le moment (e.g., contact avec le projet, installation, départ en vacances...),

b. identifié le type de dimension impliqué (une ou plusieurs parmi les 8 ci-dessus),

c. évalué la qualité d'expérience vécue sur chaque dimension significative du moment par un score synthétique sur une échelle à 5 items (de -2 (très négatif), -1 (assez négatif), 0 (neutre), +1 (assez positif), +2 (très positif). Par exemple une expérience vécue impliquant une confiance en soi très négative lors du départ en vacances valait -2 sur la dimension Confiance à ce moment précis,

d. produit un diagnostic synthétique du moment (e.g., « Peu de confiance en soi pour utiliser le bouton "Absence" lors du départ en vacances »).

28 Figure 2, point $\mathbf{D}$ - Une première évaluation quantitative synthétique a été réalisée par les chercheurs en amont du dernier recueil synthétique représenté par le point D. Durant l'évaluation, il s'est agi de faire évaluer ces moments et ces dimensions par les participants eux-mêmes du point de vue de leur propre expérience vécue et de sa qualité (cf. section 4.1). Les notes données par les participants prévalaient sur les nôtres s'il existait une différence plausible (plausibilité évaluée via les données longitudinales disponibles et le dernier entretien).

À la fin de l'entretien d'évaluation synthétique, chaque participant a été amené à se prononcer sur son souhait d'adoption ou d'abandon/rejet du système (soit après au moins deux saisons de chauffe). Ces dernières verbalisations nous ont plus particulièrement permis d'établir un diagnostic sur l'issue du processus d'appropriation en termes d'adoption ou d'abandon. Ce diagnostic a été discuté de façon contradictoire dans l'équipe d'ergonomes en regard de l'ensemble des données recueillies afin de ne pas s'appuyer uniquement sur une évaluation au temps « $t$ ». Nous considérons qu'il y avait adoption si le/la participant-e souhaitait conserver le système chez lui/elle sans réserve et si la trajectoire d'appropriation discutée dans l'équipe était cohérente par rapport à cela. Au contraire, lorsqu'une personne émettait un souhait d'adopter le système, mais avec des réserves et/ou rencontrait des difficultés d'utilisation après plusieurs années, nous avons considéré l'adoption comme réelle, mais " partielle ». Enfin, les souhaits d'abandon du système ont été considérés comme tels lorsqu'ils apparaissaient cohérents avec la trajectoire d'ensemble sur les différentes années. La construction du cadre analytique-synthétique à 3 focales 
présentées en sections 4.3 et 5 a été utile pour discuter de façon approfondie des aspects cohérents et des surprises dans les trajectoires.

Les sections suivantes présentent les enjeux du passage du point $\mathrm{D}$ à la modélisation synthétique de la complexité longitudinale de la qualité d'expérience vécue. Elles s'appuient sur un corpus final de 970 pages, caractérisant 39 moments en moyenne par foyer ( 10 foyers finalement traités après avril 2016, sur les 13 de départ). La longueur moyenne de trajectoire d'appropriation caractérisée est de 1 an et 5 mois; 3 ans au maximum et 11 mois et demi au minimum. 1308 notes ont été données lors de l'évaluation de synthèse par les participants.

\section{Un travail d'articulation de modèles se joue du recueil au traitement}

31 Avant de visualiser et discuter des graphiques de séries temporelles, le passage à une modélisation synthétique quantitative valide se joue tout d'abord dans le travail de recueil, dans celui de traitement des données et de cadrage des analyses des résultats.

\subsection{Quantifier en assurant un lien au vécu : entre traces et guidage dans l'entretien}

L'activité d'évaluation synthétique de la qualité d'expérience nous montre toute l'importance d'un travail de confrontation aux traces et de remise en situation dans les cas où plusieurs mois/années d'études sont engagés. L'usage de traces, la création de supports mnésiques et la remise en situation nous sont apparus centraux pour une quantification réaliste de la dynamique temporelle de la qualité d'expérience vécue.

La figure 3 présente un extrait des verbalisations de l'entretien d'évaluation de synthèse avec la participante 16 en avril 2016 (cf. Figure 1: icône blanc à droite, dernières évolution et évaluation de synthèse). La consigne initiale était d'évaluer la facilité d'utilisation et d'apprentissage dans les moments présentés sur la chronique, tout en verbalisant le vécu de ce moment à voix haute. Aux moments 18 et 24 (Figure 3), la participante 16 évalue la facilité d'utilisation et d'apprentissage à +2 , alors que selon nos recueils et analyses précédents, elle ne se sentait pas à l'aise avec le système. Il s'agit d'une rationalisation post-hoc. Elle s'imagine que c'était facile pour elle, car, maintenant, c'est plus facile du fait de l'appropriation qui a eu lieu en partie (notamment via l'accompagnement apporté). Nous n'intervenons pas de suite aux moments 18 et 24 afin de ne pas la placer d'emblée devant une incohérence. Ceci bien qu'elle dise «absence longue, moi je mets maintenant toujours absence longue (...) donc là c'est très bien " ce qui indique une absence d'évocation de l'expérience vécue en situation. Le moment 26 la mène vers un souvenir saillant à propos de son mail de demande d'explication sur son système à propos de ses absences courtes (« Ils ne m'ont pas répondu!»). Et, à son tour, ce souvenir engage une interprétation sur la facilité d'utilisation : «Donc, avant, c'était pas si facile ». Cette participante se rend compte que ses évaluations précédentes étaient erronées. Elle se place alors en situation par rapport à son expérience vécue au moment 24 et réévalue en conséquence. 
Figure 3 : Extrait des verbalisations d'entretien d'évaluation de synthèse avec la participante 16 en avril 2016 (cf. Figure 1 : icône blanc à droite). Elle réalise qu'elle n'éprouvait pas autant de facilité à utiliser le mode absence avant décembre 2015 et corrige ensuite les notes en conséquence.

Figure 3: Excerpt from the synthetic evaluation interview verbalisations with participant 16 in April 2016 (see Figure 1: white icon on the right). She realizes that she did not find it as easy to use the absence mode prior to December 2015 and then corrects the scores accordingly

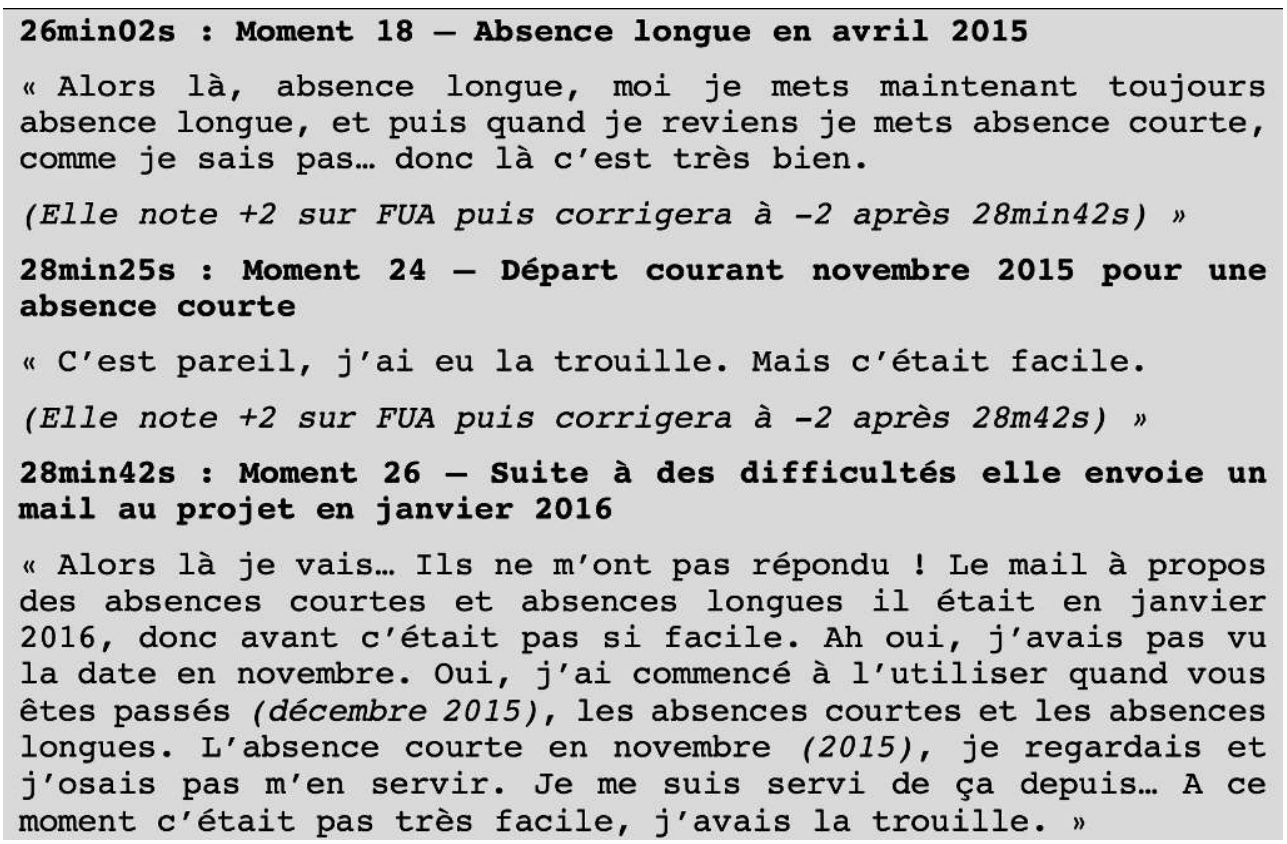

Tout entretien de remise en situation engendre des formes plurielles d'évocation de l'expérience vécue et des verbalisations qui s'en détachent. Parfois les verbalisations sont trop génériques et ne spécifient rien de l'expérience dans le moment considéré. Parfois, comme avec l'exemple ci-dessus, une incohérence évidente se manifeste entre ce qui a été dit et fait par le passé et ce qui est dit en évaluation de synthèse. Ces formes particulières de navigation entre passé imaginé ou réel, entre positions d'évocation située ou de réflexion sont à attendre dans le cas de retours sur des mois et années d'expérience. Dans ce contexte, nous relevons 6 exigences de la quantification en recueil de synthèse s'ajoutant à la nécessité générale de maîtriser les entretiens de remise en situation (e.g., explicitation, auto-confrontation) :

1. Le/la participant-e doit être amené-e dès l'introduction de la consigne à verbaliser l'expérience vécue dans le moment quantifié, quelle que soit la note,

2. Le/la participant-e doit être amené-e à ne pas quantifier si le moment et la dimension ne lui rappellent rien de significatif,

3. La chronique des moments (Figure 2, points $\mathrm{C}$ et $\mathrm{D}$ ) doit être présentée de manière à fournir un support de confrontation à son activité,

4. Ce support doit être présenté vierge de nos propres évaluations,

5. Le participant peut compléter les moments qui lui sont présentés au cours de l'évaluation par ceux qui lui reviennent en cours d'évaluation,

6. Il est important pour l'évaluateur de réaliser quelques analyses préalablement à l'évaluation de synthèse, afin de pouvoir replacer le participant-e en situation. 


\subsection{Le traitement des données quantitatives : fécondité et danger de l'« aveuglement »}

longitudinales, notre tableau de données basiques (Figure 4) contient pour chaque participant: (1) un identifiant (une feuille de calcul par participant), (2) un indicateur de temps (moments : de 1 au dernier) et (3) les valeurs des notes données pour chaque dimension à chaque moment. De plus, nous avons souhaité garder les données brutes pour pouvoir accéder à différentes focales de modélisation, pas seulement la moyenne des scores par moments par exemple. Par conséquent, nous avons gardé trace des scores négatifs, neutres et positifs à chaque moment. La feuille du tableau basique (Figure 4) contient 24 colonnes, résultant du croisement de chacune des 8 dimensions avec chaque type de valence possible: positive, neutre et négative. Si une dimension n'est pas significative vis-à-vis de l'expérience vécue sur le moment, alors il n'y a pas de note ; dans un souci de validité écologique.

Figure 4 : Extrait du tableau basique d'un participant. Pour chaque moment, les scores négatifs, neutres et positifs sont transcrits. FUA : facilité d'utilisation et d'apprentissage - EE : effectivité des économies - CT : confort thermique - AC : accompagnement - C : confiance - MS : modularité et souplesse - RF : richesse fonctionnelle - ES : esthétique.

Figure 4: Excerpt from a participant's spreadsheet. Excerpt from a participant's basic chart. For each moment, the negative, neutral and positive scores are transcribed. FUA: ease of use and learning - EE: effectiveness of savings - CT: thermal comfort - AC: support - C: trust, faith and confidence - MS: modularity and flexibility - RF: functional richness - ES: aesthetics

\begin{tabular}{|c|c|c|c|c|c|c|c|c|c|c|c|c|c|c|c|c|c|c|c|c|c|c|c|c|}
\hline \multirow{2}{*}{ Moments } & \multicolumn{8}{|c|}{ Scores négatifs } & \multicolumn{8}{|c|}{ Scores neutres } & \multicolumn{8}{|c|}{ Scores positifs } \\
\hline & $A C$ & FUA & $\mathrm{EE}$ & MS & C & CT & RF & ES & $\mathrm{AC}$ & FUA & $\mathrm{EE}$ & MS & C & $\mathrm{CT}$ & RF & ES & $A C$ & FUA & EE & MS & C & CT & $\mathrm{RF}$ & ES \\
\hline 1 & & & & & & & & & & & & & & & & & & & 2,0 & & & & & \\
\hline 2 & $-2,0$ & & $-2,0$ & & $-2,0$ & & & $-2,0$ & & & & & & & & & & & & & & & & \\
\hline 3 & $-2,0$ & & $-1,0$ & & & & & & & & & & & & & & & & & & 1,0 & & & \\
\hline $3 b$ & & & & & $-2,0$ & & & & & & & & & & & & & & & & & & & \\
\hline 4 & $-1,0$ & & & $-1,0$ & & & & & & & 0,0 & & & & & & & & & & & & & \\
\hline 5 & & & & & & & $-1,0$ & & & & 0,0 & & & & & & & 2,0 & & & & & 2,0 & \\
\hline
\end{tabular}

Ce moment et celui des premiers traitements et discussions de séries temporelles constituent un "aveuglement", au sens où s'y joue la césure entre l'analyse de l'expérience vécue et la synthèse quantitative liée aux notes données sur la qualité d'expérience vécue. Cette césure a lieu du fait de l'activité de traitement actif des données quantitatives, impliquant un temps et une façon de penser qui diffèrent de l'analyse qualitative initiale. En un sens, cet aveuglement est nécessaire et potentiellement fécond, car la césure permet ensuite de confronter les diagnostics qualitatifs et quantitatifs, analytiques et synthétiques; de se laisser surprendre par les formes d'évolution visualisées une fois les données traitées correctement. En un autre sens, il est classiquement dangereux au sens où il pourrait permettre toutes les interprétations s'il n'était pas ensuite rapporté à l'expérience vécue grâce aux verbatims et aux analyses qualitatives préalables de 2014 et 2015. 


\subsection{Un cadre analytique-synthétique à 3 focales}

38

Comme le dévoile la section suivante, nous avons finalement développé un cadre à 3 focales sur la qualité de l'expérience vécue. Une focale est le regroupement de plusieurs éléments de modélisation: une échelle temporelle (un moment, plusieurs moments ou la série temporelle de tous les moments), un type d'analyse (qualitative ou quantitative), une étendue de dimensions (une dimension en particulier ou toutes), et un type de traitement quantitatif (notes ajustées brutes, moyenne ou tendance par régression linéaire).

Les différentes focales sont numérotées de 1 à 3 caractérisant des niveaux progressifs de synthèse et abstraction. La focale qualitative locale $\left(n^{\circ} \mathbf{1}\right)$ est construite à partir des verbalisations des acteurs sur les moments. Elle fournit la base de construction des autres focales et nous permet des diagnostics qualitatifs sur l'expérience vécue dans ces moments et sur l'appropriation du système. La focale quantitative radiale $\left(n^{\circ} 2\right)$ est construite à partir du modèle radial à deux pôles (Figure 7) permettant d'apprécier la valence positive/négative de l'expérience sur les dimensions, et sa valeur ( 0,1 ou 2$)$. Elle donne accès à la structure de la qualité de l'expérience vécue quantifiée du moment, ou à une série temporelle partielle de ces moments. La focale quantitative sérielle $\left(\mathbf{n}^{\circ} \mathbf{3}\right)$ est construite à partir d'une ou plusieurs séries temporelles complètes (Figures 5 et 6 ) des notes ajustées brutes. Elle peut concerner une seule dimension (elle est dite dimensionnelle dans ce cas) ou toutes à chaque moment (elle est dite générale). Elle s'accompagne de la tendance par régression linéaire sur tous les moments. Cette focale apporte un éclairage général sur la dynamique de qualité de l'expérience vécue sur plusieurs années.

Une série temporelle représente l'évolution d'une suite finie d'états/quantités sur un axe chronologique. Les séries temporelles sont des modèles statistiques de complexité minimale (Hilpert \& Marchand, 2018). Elles permettent cependant de visualiser des dynamiques longitudinales différemment dans notre projet. La construction de notre «métrique sensée du temps » (Singer \& Willett, 2003) est basée sur le point de vue de l'acteur et sa propre construction de significations pendant les moments d'appropriation (par exemple, le moment de l'installation, celui du départ en vacances, etc.). Par conséquent, si l'intervalle temporel entre chaque moment d'appropriation est représenté de façon homogène pour faciliter la lecture (e.g. Figure 5) les durées chronologiques des intervalles diffèrent puisqu'elles dépendent des moments où l'expérience a eu lieu. L'intervalle peut être un jour comme un mois. D'après Singer et Willett (2003) ceci relève d'un travail sur des données temporelles dites "nonstructurées $»^{3}$. Par ailleurs, nos séries temporelles ne sont pleinement légitimes qu'à partir du moment on l'on crédite l'hypothèse d'une continuité de significations pour l'acteur à travers le temps long, comme c'est le cas pour l'objet théorique cours de vie relatif à une pratique (Theureau, 2006) par exemple. Enfin, l'objectif assigné à ces séries temporelles est relativement modeste : permettre de traiter d'autres aspects de la dynamique d'appropriation que l'analyse qualitative ne nous aurait pas permis de voir. 


\section{Utilité des focales et de leur articulation}

\subsection{Focale 3 générale : comparer les dynamiques longitudinales}

41 L'utilité première de la focale 3 générale est de mettre à plat les dynamiques longitudinales de chaque foyer sur toute la durée du recueil. Malgré ses limites évidentes liées à un traitement par la moyenne générale des scores sur chaque moment, elle permet de comparer visuellement les trajectoires les unes aux autres, ce qui facilite une prise de recul générale sur les données traitées. Par exemple, la Figure 5 montre avec évidence que certaines trajectoires relèvent d'une qualité de vécu globalement positif à travers le temps (participant 11), là où d'autres relèvent de diagnostics plus mitigés (participant 16) ou négatifs (participant 15).

Couplée aux diagnostics issus de la focale qualitative locale $\left(n^{\circ} 1\right)$, elle permet aussi d'initier des discussions sur les liens entre la valence de l'expérience (positive ou négative) et la trajectoire d'appropriation; ou bien sur la valeur de chaque moment dans cette trajectoire. Par exemple, on voit ici que toutes les séries temporelles varient fortement entre valences négatives et positives à travers le temps. Cela signifie que les participants ont tous vécu plusieurs moments très négatifs et très positifs. Par ailleurs on voit aussi que ceci est vrai dans le cas de trajectoire ouvrant sur une adoption comme sur un rejet à la fin du projet (focale qualitative locale $\left(n^{\circ} 1\right)$. Par conséquent, il n'existe pas de relation simple et directe entre la valence de la qualité de l'expérience vécue par l'utilisateur et son adoption du système.

Il n'existe pas non plus de relation simple avec le statut négatif ou positif de la valence et l'intégration du système. La focale qualitative locale $\left(\mathrm{n}^{\circ} 1\right)$ nous a montré à plusieurs reprises que certaines expériences vécues négativement donnent lieu à apprentissages et à échanges, favorisant l'intégration du système au monde et à la culture propre de l'utilisateur. Au moment d'une panne ou d'un dépannage par exemple, les participants peuvent être surpris négativement, mais bien pris en charge par le service d'assistance. Ils peuvent alors apprendre de nouvelles choses à cette occasion (e.g. comment fonctionne une partie du système) qui vont ensuite structurer d'autres moments d'appropriation. 
Figure 5 : Groupement de représentations des dynamiques longitudinales par la série temporelle en focale 3-générale, afin de faciliter la comparaison entre participants. Les ronds noirs correspondent au score moyen obtenu sur toutes les dimensions significatives d'un moment. La ligne noire est la régression linéaire des moyennes de toutes les dimensions.

Figure 5: Grouping of representations of the longitudinal dynamics by the time series in 3-general focus, in order to facilitate the comparison between participants. The black circles correspond to the average score obtained on all the significant dimensions of a moment. The black line is the linear regression of the averages of all the dimensions

Focale 3 - générale

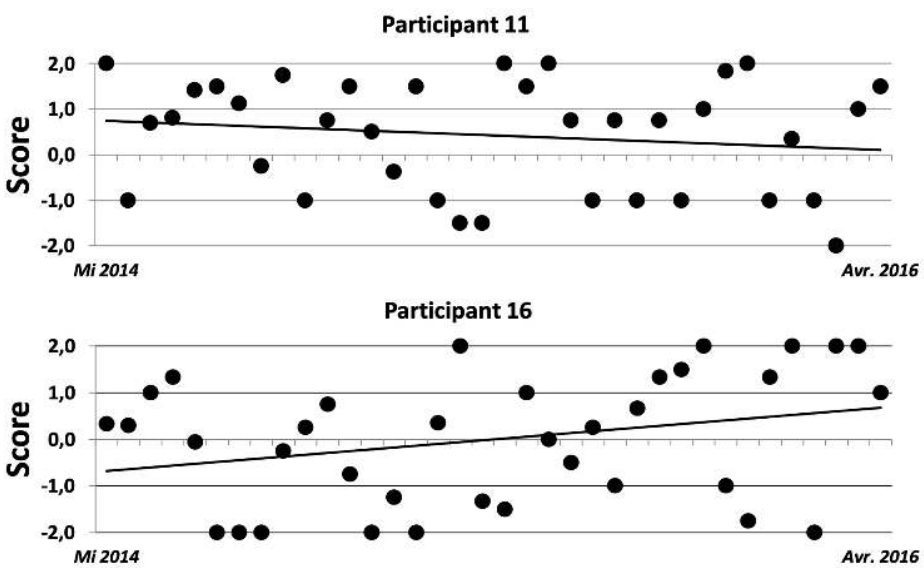

Participant 15

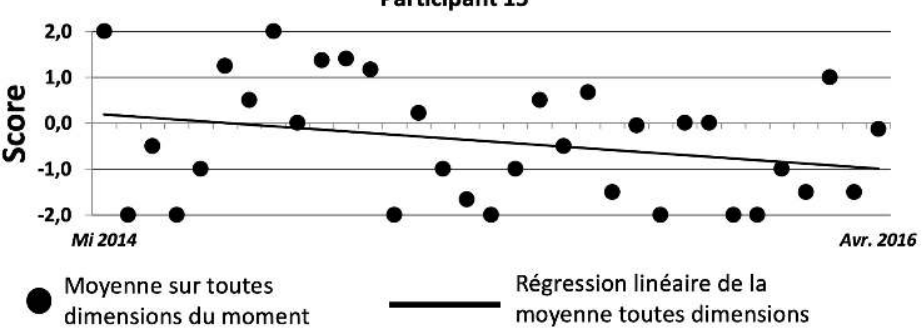

Diagnostics - Focale 1

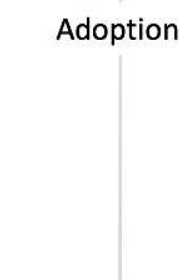

Adoption partielle

Rejet peut se demander comment une dynamique générale qui tend vers un score moyen plus négatif ou nul au fil de l'étude peut correspondre à une adoption. Devrait-on anticiper un abandon possible dans les années à venir et pour quelles raisons? Une dimension joue-t-elle un rôle plus important que les autres? La question est inversée pour la participante 16: comment est-il possible de partir d'un pôle aussi négatif d'expérience pour aboutir à une adoption partielle?

À partir de ce type de constat, deux optiques de traitements plus avancés paraissent intéressantes: un traitement affinant les aspects statistiques et l'autre précisant les articulations possibles de la focale 3 quantitative sérielle aux significations construites en situation (focale 1 qualitative locale) pour expliquer ces scores qualitativement. Nous présentons cette seconde optique dans les deux sous-sections suivantes et reviendrons sur la première en discussion.

\subsection{Focale 3-dimensionnelle : caractériser les moments et dimensions clés}

Du point de vue de l'expérience vécue, toutes les dimensions et tous les moments ne se valent pas. Certains sont "clés» et d'autres non. Du point de vue de la focale qualitative locale $\left(n^{\circ} 1\right)$, une dimension clé renvoie à une préoccupation/attente très 
forte du participant, de telle sorte qu'elle prévaut sur les autres. Sur le plan statistique, une dimension clé accentue la tendance de la moyenne générale, tout en étant cohérente avec le diagnostic qualitatif de la focale qualitative locale $\left(n^{\circ} 1\right)$. L'effectivité des économies (EE) pour le participant 15 présenté sur la Figure 6 ci-dessous correspond à une dimension clé.

Figure 6 : Séries temporelles quantitatives sérielles générale (Ronds noirs et droite continue) et dimensionnelle pour l'Effectivité des économies (EE - carrés gris et droite en petits pointillés). En abscisses : la succession temporelle des moments. En ordonnées : le score de l'évaluation de la qualité d'expérience vécue. Cette série couvre 34 moments allant de mi-2014 à avril 2016 pour le participant 15.

Figure 6: General quantitative time series (black circles and continuous straight line) and dimensional time series for Economy Effectiveness (EE - grey squares and dotted straight line). On the x-axis: the temporal succession of moments. $Y$-axis: the quality of experience evaluation score. This series covers 34 moments from mid-2014 to April 2016 for participant 15

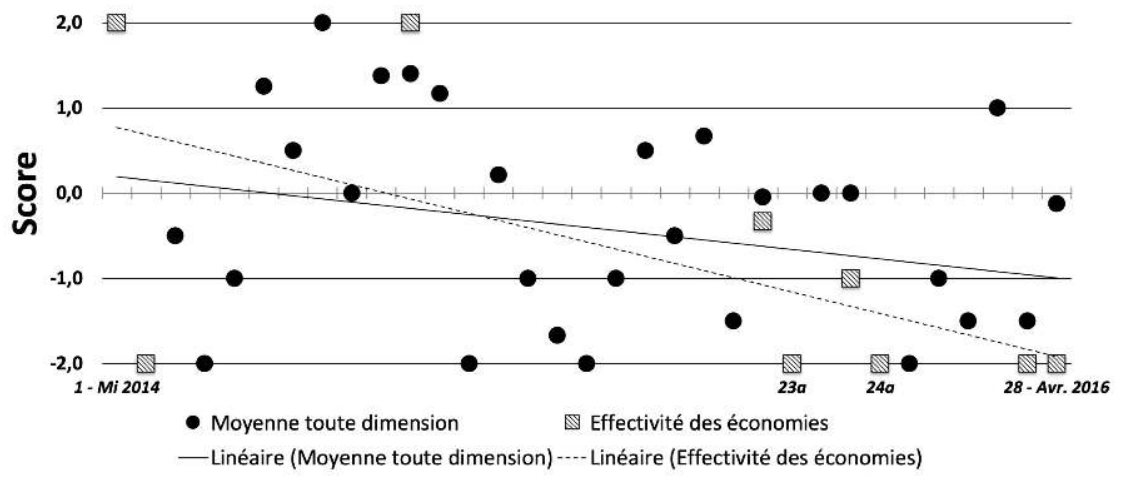
1 - Mi-2014 - EE =+2 : "C'était le but, possibilité de savoir les économies, Oui, là
je veux maîtriser mon chauffage. Je souhaite être pro-actif."

23a - Mai 2015 - EE\& C = - 2 : "Depuis qu'on a mis ce système en place, on consomme plus qu'avant. J'ai reçu des infos."

24a - Juil. 2015 - EE \& C = -2 : " Je me suis rendu compte par hasard, un an après, que les chauffe-eau étaient en train de chauffer en pleine journée. "

28 - Avr. $2016-\mathrm{EE}=-2 / \mathrm{C}=-2$; "Ne pas pouvoir évaluer et en plus ne pas économiser, ça ça fait doublement mal. Je suis pas sûr du tout, j'ai pas de visibilité "

Au plan statistique sur la figure 6, la droite de régression d'EE présente un coefficient plus négatif que la droite de la moyenne de la focale 3 générale; le plus négatif de toutes les dimensions par ailleurs. Elle indique donc une dynamique de plus grande amplitude du début à la fin, en direction du négatif. Qui plus est, on voit avec les verbatims de la focale qualitative locale $\left(n^{\circ} 1\right)$ à quel point EE comptait pour le participant 15. La dimension EE est clé pour ce participant. Elle renvoie en fait à un ensemble d'ouverts (Theureau, 2006) et d'attentes fortement structurantes pour la dynamique longitudinale du participant 15 : connaître les économies, mieux maîtriser mon chauffage, être pro-actif. Elles sont une partie du «monde propre» de ce participant. En matière d'intégration, on voit aussi que ces ouverts n'entrent pas en "collision" avec une expérience critique du système avant le mois de mai 2015 (moment 23a), soit près d'un an après le début de la trajectoire à mi-2014. De telle sorte que durant environ un an, des expériences positives et négatives sont vécues autour du système et l'intégration se déroule, mais cela n'engage rien de vraiment décisif concernant l'adoption du système. Au moment 23a, l'intégration du système à l'expérience vécue du participant a lieu sur un mode très négatif puisqu'il constate une 
surconsommation alors qu'il attendait de connaître les économies et de mieux maîtriser son chauffage notamment. Ensuite, le système s'intègre à nouveau à cet ensemble de façon très négative (24a et 28 notamment) dans l'expérience vécue, menant à un rejet à la fin de notre évaluation.

Cette focale, toujours articulée à la focale qualitative locale $\left(n^{\circ} 1\right)$, nous a permis de préciser le rôle de chaque dimension de l'expérience dans la dynamique d'appropriation de chaque participant. Par exemple, l'effectivité des économies, lorsqu'elle est clé, amène l'acteur à ne pas être impacté de façon critique dans les premiers moments d'appropriation pour les raisons explicitées ci-dessus. L'effet premier d'une préoccupation-attente clé liée à $\mathrm{EE}$ est de faire tenir l'usage et les attentes sur le long terme avant une expérience ou plusieurs qui sont vraiment décisives. De ce fait, on est autorisé à synthétiser la trajectoire en regard de cette dimension et des moments clés qui la mettent en jeu au plan de l'expérience vécue, si celle-ci présente bien la dimension comme étant significative. En revanche, tous les foyers ne partagent pas les mêmes préoccupations et attentes de départ. De ce fait, l'importance des premiers moments d'appropriation (e.g. prise en main) peut être plus grande pour des participants attendant surtout de la facilité d'utilisation (FUA) et/ou n'ayant pas de préoccupation forte quant à l'effectivité des économies. Des foyers participants ont ainsi stoppé leurs utilisation et découverte du système lors de la prise en main (début de trajectoire), ne comprenant pas comment l'utiliser et n'ayant pas d'ouvert structurant qui aurait permis de faire persister une forme d'appropriation et utilisation.

Enfin, le caractère clé de certaines dimensions explique en partie pourquoi le participant 11 adopte le système installé chez lui alors que globalement, la qualité d'expérience tend vers 0 ou le négatif avec la focale 3 quantitative sérielle (Figure 5). Le système s'intègre correctement à ses dimensions clés (modularité et souplesse notamment) et les autres dimensions sont de moindre importance. Mais comme notre modèle statistique ne pondère pas les scores en fonction du caractère clé d'une dimension, des dimensions "non-clés » peuvent faire tendre la trajectoire vers le négatif alors que la qualité d'expérience est suffisamment bonne pour ouvrir sur une adoption du système. D'où l'importance d'une analyse à plusieurs focales dans un premier temps.

\subsection{Focale quantitative radiale $\left(n^{\circ} 2\right)$ : rendre compte de la structure dimensionnelle des moments}

À l'(in)stabilité temporelle de la dynamique de qualité d'expérience vécue, la focale quantitative radiale $\left(n^{\circ} 2\right)$ ajoute l'angle situationnel. Non seulement la qualité fluctue fortement au cours du temps entre valences positive, neutre et négative, mais les dimensions impliquées varient fortement elles aussi. Ainsi, aucun des moments présentés sur la Figure 7 ci-dessous ne possède la même structure dimensionnelle. Cela signifie que dans l'expérience vécue, ces dimensions ne se présentent jamais de la même façon entre différents moments. Parfois un moment est vécu uniquement sur une dimension, parfois sur plusieurs... Sans pouvoir affirmer avec certitude qu'aucun moment n'est absolument le même, on voit sans aucun doute que la structure dimensionnelle des moments varie fortement d'un moment à l'autre. 
Figure 7 : Synthèse empirique de la dynamique longitudinale d'appropriation du système par le participant 15 , sur la base de la focale quantitative radiale $\left(n^{\circ} 2\right)$ et de diagnostics locaux issus de la focale qualitative locale $\left(n^{\circ} 1\right)$. FUA : facilité d'utilisation et d'apprentissage - EE : effectivité des économies - CT : confort thermique - AC : accompagnement - C : confiance - MS : modularité et souplesse - RF : richesse fonctionnelle - ES : esthétique.

Figure 7: Empirical synthesis of the longitudinal dynamics of appropriation of the system by participant 15 , based on the radial quantitative focus $\left(n^{\circ} 2\right)$ and local diagnoses from the local qualitative focus $\left(n^{\circ} 1\right)$. FUA: ease of use and learning - EE: effectiveness of savings - CT: thermal comfort - AC: support - C: trust, faith and confidence - MS: modularity and flexibility - RF: functional richness - ES: aesthetics

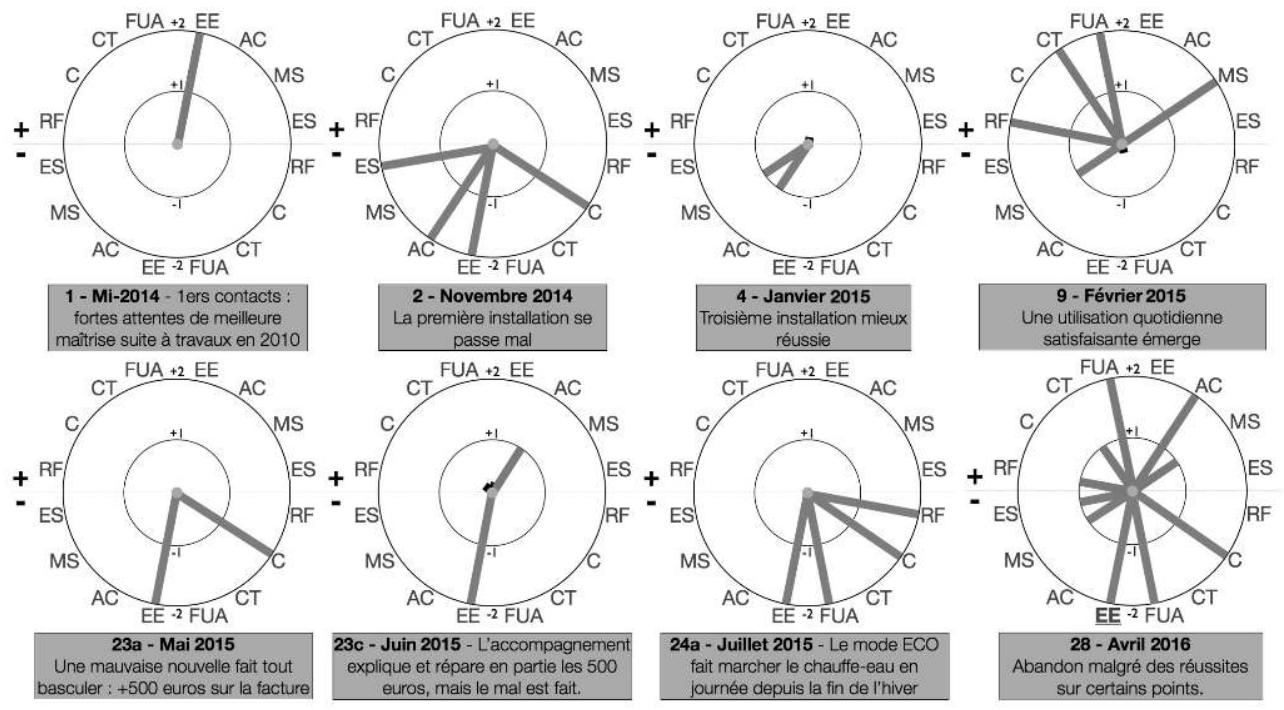

51 Cette focale nous aura été utile pour visualiser et faire visualiser synthétiquement l'évolution de la dynamique sur plusieurs années, à partir d'un nombre restreint de moments (8 ici). Cette sélection doit être représentative des moments clés (ici, 1, 23a, 23c, 24a), mais aussi des moments rendant compte de la dynamique intermédiaire. Par exemple, le moment 9 ci-dessus montre bien qu'une utilisation quotidienne a existé pour le participant 15, malgré la dynamique de rejet qui émergera par la suite. On notera aussi que ce moment 9 n'inclue pas de vécu sur la dimension clé $\mathrm{EE}$ : il est donc hélas peu déterminant. À l'inverse, les moments 2 et 4 montrent bien que des expériences négatives au début, pour ce participant, n'engagent pas de suite un rejet.

Cette focale aura aussi permis de créer un objet intermédiaire sur tablette tactile permettant aux acteurs du projet n'ayant pas connaissance de ces dynamiques temporelles de les explorer librement eux-mêmes tout en les discutant avec d'autres. Cet objet présente nos diagnostics locaux sur les 8 moments plus des verbatims donnant du sens aux scores visibles sur les radars. En plus des pistes de conception de 2015, il a constitué un modèle pratique (Theureau, 2006) final, empiriquement étayé sur la démarche précédente.

\section{Discussion}

53 Nous nous demandions ce que l'on pouvait attendre d'une articulation entre modèles analytique et synthétique (et les méthodes qui les accompagnent) pour la compréhension des dynamiques longitudinales d'activité individuelle. La section 4 démontre tout d'abord que cette articulation implique un travail permettant de: (1) quantifier en assurant un lien à l'expérience vécue ; (2) maîtriser l'équilibre entre 
fécondité et danger de l'«aveuglement» du traitement quantitatif; (3) articuler plusieurs focales d'analyse-synthèse.

À ce titre, notre article propose un cadre de travail dont nous espérons qu'il ouvre des perspectives pour de futures approches longitudinales de l'expérience vécue et/ou de l'activité individuelle-sociale. Bien qu'issu d'une recherche longitudinale, ce cadre peut tout à fait être adapté à des contextes de recherche et d'intervention plus restreints temporellement, en réduisant les nombres de recueils et en ciblant plus précisément les moments à évaluer (cf. ci-dessous).

La section 5 a montré que cette articulation permet à sa façon de: (1) renforcer l'analyse qualitative (qui reste essentielle et fondamentale); (2) préciser les données quantitatives par l'analyse de l'expérience vécue en situation(génériciser des dynamiques sur des temps très étendus en limitant les risques de l'aveuglement ou de l'interprétation abusive de données); (3) visualiser plus facilement les dynamiques de long terme et les situer; (4) susciter de nouvelles questions, étayer de nouvelles hypothèses; (5) faciliter l'engagement d'une démarche plus déductive en complément de l'induction/abduction privilégiée dans l'analyse qualitative.

Sur ce dernier point, nous avons vu que si des attentes fortes existent sur la dimension d'Éffectivité des Économies, alors on peut s'attendre à ce que les premiers moments de la trajectoire ne déterminent pas l'adoption/rejet; on doit attendre au moins deux saisons de chauffe pour voir apparaitre une forme de moment décisif. S'il n'existe pas d'attente forte sur cette dimension, alors d'autres dimensions peuvent impacter de façon décisive l'appropriation, mais cela dès les premiers instants (pas après 2 saisons de chauffe) : par exemple la facilité d'utilisation et d'apprentissage du système ou l'accompagnement. Ce qui importe dans notre étude, étant donné le stade initial de réflexion, est plus la possibilité d'élaborer ces formes d'hypothèses que leur seule validité, puisque cela démontre l'intérêt d'une articulation analytique-synthétique pour élaborer de nouvelles hypothèses et entrer dans une démarche plus déductive. D'un point de vue scientifique il serait d'ailleurs plus intéressant de constater leur invalidité que de les confirmer en totalité.

Ce type de résultat et d'hypothèse pourrait aussi changer la façon dont on envisage le recueil des données dans les études sur l'appropriation. Singer \& Willett (2003) considèrent qu'il est parfois possible et intéressant de recueillir plus de données dans les moments critiques. Encore faut-il disposer d'une théorie sur ce qu'est un moment critique vis-à-vis du domaine de connaissance. Dans notre étude, dans le cas d'attentes fortes sur EE, les moments critiques se trouvent à au moins deux saisons de chauffe après l'installation du système. Dans le cas d'absence d'attentes fortes sur cette dimension, le début de la trajectoire (installation, prise en main) peut s'avérer critique et justifier des recueils plus nombreux ou plus précis. Au début de notre étude, les 39 moments étudiés par participants n'avaient pas de statuts distinctifs. De telles théories, si elles doivent toujours être appréciées de façon critique, auraient permis d'organiser différemment la recherche longitudinale.

Une limite importante des modèles synthétiques présentés dans cet article est leur absence d'apport supplémentaire de recommandation à la conception des systèmes et situations de gestion d'énergie (en plus du temps de recueil et traitement demandé dans notre recherche, mais celui-ci peut être réduit drastiquement pour des interventions usuelles en ergonomie ou UX research). Ceci est logique puisque ces modèles engagent des contenus plus génériques, abstraits et dé-situés de l'activité 
humaine. Ici, l'analyse qualitative de l'activité en situation reste l'approche la plus pertinente. En revanche, appliquer de telles méthodes synthétiques sur une population beaucoup plus large permettrait certainement de caractériser des patterns de stabilité et d'instabilité (e.g. Harbich \& Hassenzahl, 2017) dans les trajectoires d'appropriation de façon plus conséquente ; et donc peut-être de guider plus précisément les projets de conception pour un passage à l'échelle par exemple. Un autre usage possible de ce travail pourrait être la conception d'outils informatiques plus avancés pour la caractérisation ou la modélisation de telles trajectoires.

Ces éléments nous semblent donc, même très modestement à ce stade, ouvrir vers un début de mathématisation possible et un dialogue en partie nouveau entre sciences de la complexité et approches de l'activité humaine. Ces efforts nécessiteraient cependant, une mise en vis-à-vis plus ferme, critique et explicite des notions et concepts de chaque " monde "; chose qui, jusqu'à maintenant, n'a été faite qu'itérativement dans notre travail. Par exemple nous nous interrogeons sur les liens et les contours des notions d'« ouvert/préoccupation » (issue du cours d'action), « dimension clé » (issue de notre travail intermédiaire, cf. section 5.2) et d'« attracteur » en science de la complexité. Les " changements profonds et durables dans l'état d'un système [...] correspondent à des changements dans la structure des attracteurs" (Wiese et al., 2010, p. 1027). La dynamique longitudinale d'appropriation dévoilée en section 5.2 est très fortement structurée par l'ouvert "mieux maîtriser mon chauffage, savoir combien je consomme ", si bien qu'il semble réguler l'importance des moments d'appropriation pour le participant 15 et qu'il nécessiterait de pondérer à la hausse la dimension EE sur le plan statistique dans la série temporelle. Dans ce cadre, cet ouvert devrait-il être envisagé comme un attracteur de phase du couplage structurel entre le participant 15 et son système de gestion d'énergie? Comme un attracteur dans la structure des dimensions? Pas du tout comme un attracteur? Il en va de même pour la relation entre les notions de situation (au sens du cours d'action), de moment (dans notre étude) et de vecteurs (sciences de la complexité) par exemple.

Par conséquent, l'idéal d'articulation analytique-synthétique proposé par Theureau $(2005,2006)$ paraît tout à fait pertinent en ce qui concerne la complexité longitudinale de l'activité humaine, mais nécessitera encore de nombreux apports pour dépasser les limites actuelles. Il appellerait la poursuite d'efforts d'articulation entre les approches de la complexité temporelle : l'une ancrée dans une phénoménologie de l'activité et/ou de l'expérience vécue, l'autre ancrée dans les concepts et modèles issus des systèmes complexes. Avec, au milieu, des hypothèses intermédiaires, périphériques ou supplémentaires, comme celles de l'activité individuelle comme système complexe par exemple, ou celle de la qualité d'expérience vécue.

61 Enfin nous nous interrogeons sur la contribution possible de ce travail à d'autres domaines convoquant le temps long en ergonomie, soulignés en introduction (santé et maladies professionnelles, gestion temporelle, formation, soutenabilité, etc.). Mardon et al. (2013) concluent leur analyse diachronique quantitative des TMS en soulignant qu'il est nécessaire d'articuler des résultats numériques avec l'analyse de l'activité, ainsi qu'avec des entretiens à visée rétrospective. Notre travail va pleinement en ce sens, en proposant une façon de considérer la «subjectivité » dans l'activité de façon dynamique et longitudinale: entre entretiens de remise en situation centrés sur l'expérience vécue, quantification située, analyse qualitative et synthèse quantitative. Ce travail ouvre aussi des perspectives pour la caractérisation de moments de bascule 
dans les trajectoires d'activité individuelle (moments clés, etc.), ainsi que pour reconsidérer l'importance à accorder à différentes structures cognitives/émotionnelles (e.g. préoccupations, attentes, etc.) dans l'évolution de long terme de l'activité. Il semblerait donc intéressant d'approfondir les discussions croisées entre différentes approches des temps longs et temporalités multiples de l'activité humaine.

\section{BIBLIOGRAPHIE}

Albaladejo, C., Magda, D., Geslin, P., \& Salembier, P. (2009). La mise à l'épreuve : le transfert des connaissances scientifiques en questions. Quae.

Amalberti, R., Montmollin, M. de, \& Theureau, J. (Eds.). (1991). Modèles en analyse du travail. Editions Mardaga.

Badiou, A. (1969). Le concept de modèle (Éd. 2007). Fayard.

Barbier, C., Cerf, M., \& Lusson, J.-M. (2015). Cours de vie d'agriculteurs allant vers l'économie en intrants : Les plaisirs associés aux changements de pratiques. Activités, 12(2). https://doi.org/ $10.4000 /$ activites. 1081

Bationo-Tillon, A., Folcher, V., \& Rabardel, P. (2010). Les instruments transitionnels : Une proposition pour étudier la diachronie des activités narratives. Activités, 7(2). https://doi.org/ 10.4000/activites.2437

Boeing, G. (2016). Visual Analysis of Nonlinear Dynamical Systems: Chaos, Fractals, Self-Similarity and the Limits of Prediction. Systems, 4, 37.

Bourbousson, J. (2013). Cours d'action et données quantitatives : L'analyse de l'activité collective. eJRIEPS. Ejournal de la recherche sur l'intervention en éducation physique et sport, 30. http:// journals.openedition.org/ejrieps/2473

Cahour, B., Salembier, P., \& Zouinar, M. (2016). Analyzing lived experience of activity. Le Travail Humain, 79(3), 259-284.

Chizallet, M., Prost, L., \& Barcellini, F. (2019). Comprendre l'activité de conception d'agriculteurs en transition agroécologique : Vers un modèle trilogique de la conception. Psychologie Française, 64(2), 119-139. https://doi.org/10.1016/j.psfr.2019.03.001

Daniellou, F. (2005). The French-speaking ergonomists' approach to work activity: Crossinfluences of field intervention and conceptual models. Theoretical Issues in Ergonomics Science, 6(5), 409-427. https://doi.org/10.1080/14639220500078252

Fréjus, M. (2019). Élargissement et renouvellement des questions traitées par l'ergonomie dans le domaine du développement durable : Retour sur 12 ans de travaux sur les activités domestiques et la maîtrise des consommations énergétiques. Psychologie Française, 64(2), 179-196. https:// doi.org/10.1016/j.psfr.2018.07.002

Guastello, S. J. (2017). Nonlinear dynamical systems for theory and research in ergonomics. Ergonomics, 60(2), 167-193. https://doi.org/10.1080/00140139.2016.1162851 
Guibourdenche, J., Poret, C., Poizat, G., Motté, F., Haradji, Y., Salembier, P., \& Galbat, M. (2019). Sustainable Development and Energy Systems Design: Issues and Perspectives from a Francophone Activity-Centered Approach. In A. Thatcher, K. J. Zink, \& K. Fischer (Eds.), Human Factors for Sustainability (p. 329-357). Taylor \& Francis.

Haradji, Y., Guibourdenche, J., Reynaud, Q., Poizat, G., Sabouret, N., Sempé, F., Huraux, T., \& Galbat, M. (2018). De la modélisation de l'activité humaine à la modélisation pour la simulation sociale : Entre réalisme et fécondité technologique. Activités, 15(1). https://doi.org/10.4000/ activites.3106

Harbich, S., \& Hassenzahl, M. (2017). User Experience in the Work Domain: A Longitudinal Field Study. Interacting with Computers, 29(3), 306-324. https://doi.org/10.1093/iwc/iww022

Haué, J.-B. (2003). Conception d'interfaces grand public en termes de situations d'utilisation. Le cas du "Multi-Accès ». Thèse de contrôle des systèmes, non-publiée. Université Technologique de Compiègne.

Hilpert, J. C., \& Marchand, G. C. (2018). Complex Systems Research in Educational Psychology: Aligning Theory and Method. Educational Psychologist, 53(3), 185-202. https://doi.org/ 10.1080/00461520.2018.1469411

Karapanos, E., Martens, J.-B., \& Hassenzahl, M. (2012). Reconstructing experiences with iScale. International Journal of Human-Computer Studies, 70(11), 849-865. https://doi.org/10.1016/j.ijhcs. 2012.06.004

Kujala, S., Roto, V., Väänänen-Vainio-Mattila, K., Karapanos, E., \& Sinnelä, A. (2011). UX Curve: A method for evaluating long-term user experience. Interacting with Computers, 23(5), 473-483. https://doi.org/10.1016/j.intcom.2011.06.005

Mardon, C., Buchmann, W., \& Volkoff, S. (2013). Un enfoque diacrónico de los TME : Uso de datos cuantitativos en una gran empresa. Laboreal, 9(1). https://doi.org/10.4000/laboreal.6085

Singer, J. D., \& Willett, J. B. (2003). Applied Longitudinal Data Analysis: Modeling Change and Event Occurrence. Oxford University Press, USA.

Thatcher, A. (2016). Longevity in a sustainable human factors and ergonomics system-of-systems. 22a SEMANA DE LA SALUD OCUPACIONAL. https://www.researchgate.net/publication/ 309606212_Longevity_in_a_sustainable_human_factors_and_ergonomics_system-of-systems

Thatcher, A., Guibourdenche, J., \& Cahour, B. (2019). Sustainable system-of-systems and francophone activity-centered approaches in ergonomics: Converging and diverging lines of dialogue. Psychologie Française, 64(2), 159-177. https://doi.org/10.1016/j.psfr.2018.07.001

Theureau, J. (1992). Le cours d'action: Analyse sémio-logique. Essai d'une anthropologie cognitive située. Peter Lang.

Theureau, J. (2005). Les principes de la connaissance scientifique de la complexité technicoorganisationnelle selon le programme de recherche "cours d'action" en dix-sept questions. http:// www.coursdaction.fr/08-nonpublies/2005-JT-T22.pdf

Theureau, J. (2006). Le cours d'action: Méthode développée. Octarès.

Theureau, J. (2010). Les entretiens d'autoconfrontation et de remise en situation par les traces matérielles et le programme de recherche « cours d'action ». Revue d'anthropologie des connaissances, 4(2), 287-322. 
Walker, G. H., Stanton, N. A., Salmon, P. M., Jenkins, D. P., \& Rafferty, L. (2010). Translating concepts of complexity to the field of ergonomics. Ergonomics, 53(10), 1175-1186. https://doi.org/ $10.1080 / 00140139.2010 .513453$

Wiese, S. L., Vallacher, R. R., \& Strawinska, U. (2010). Dynamic Social Psychology: Complexity and Coherence in Human Experience. Social and Personality Psychology Compass, 4(11), 1018-1030. https://doi.org/10.1111/j.1751-9004.2010.00319.x

Wisner, A. (1972). Diagnosis in ergonomics: The choice of operating models in field research. Ergonomics, 15(6), 601-620. https://doi.org/10.1080/00140137208924462

Zara-Meylan, V. (2013). Modalidades de gestão do meio temporal em uma condução de processos múltiplos em situação dinâmica : Uma pesquisa em empresas de horticultura. Laboreal, 9(1), 60-65.

Zara-Meylan, V. (2016). Quelles conceptions temporelles pour analyser l'activité ? Une proposition issue de recherches en ergonomie dans l'horticulture. Activités, 13(1). https:// doi.org/10.4000/activites.2732

\section{NOTES}

1. Le terme "moment d'appropriation" est utilisé ici pour caractériser opérationnellement l'appropriation. L'appropriation étant un processus, nous ne prétendons pas rendre compte de sa totalité avec une suite de moments, mais nous mobilisons cette suite de moments comme base empirique de référence. La transformation des connaissances ou des modes d'utilisation du système entre deux moments permet de déterminer en quoi un moment particulier joue dans l'appropriation (cf. Figure 5).

2. Au contraire d'une application stricte d'une grille de catégories extrinsèques et prédéfinies.

3. Cf. Singer et Willett (2003, pp. 11-12) : « La seule contrainte est que, comme le temps lui-même, la variable temporelle ne peut changer que de façon monotone, - en d'autres termes, elle ne peut pas changer de direction. (...) Si tout le monde est évalué selon un calendrier identique, on dit que l'ensemble des données est structuré dans le temps. Si les calendriers de collecte des données varient d'un individu à l'autre, nous disons que l'ensemble de données est non structuré dans le temps. La modélisation de l'évolution individuelle est suffisamment flexible pour gérer les deux possibilités.». Puis ils poursuivent: "Vous pouvez facilement construire un ensemble de données par personne et par période, même si chaque participant a un calendrier de collecte de données unique. (...) Une variable temporelle dédiée permet également aux ensembles de données «personnes-période » de s'adapter à des modèles de recherche dans lesquels le nombre de mesures varie d'une personne à l'autre.» (p. 23) (Notre traduction)

\section{RÉSUMÉS}

Un problème récurrent des recherches longitudinales sur l'activité humaine est leur absence d'articulation entre modèle analytique qualitatif et modèle synthétique quantitatif d'évolution dynamique. Cette absence engendre des difficultés dans la justification des choix d'analyse et de synthèse et empêche d'aboutir à une démarche déductive sur les dynamiques longitudinales. Dans le cadre d'une recherche empirique et technologique longitudinale de 3 ans sur 
l'appropriation, cet article discute des conditions de production et de l'utilité d'une modélisation synthétique quantitative de la complexité longitudinale de l'activité individuelle, en articulation avec une méthode analytique qualitative. Plus que la mobilisation d'un modèle seul et pré-donné, le travail de modélisation relève d'une articulation active entre méthodes-modèles analytiques (classiques en analyse d'activité) et méthodes-modèles synthétiques. Cette articulation offre des perspectives d'amélioration des études longitudinales centrées sur l'activité humaine et fournit des supports visuels utiles en projet de conception. Nous discutons des apports et limites de ce travail vis-à-vis de l'analyse de l'activité en ergonomie de langue française et des sciences de la complexité plus souvent mobilisées dans les facteurs humains.

One recurring problem in longitudinal research on human activity is the lack of articulation between qualitative analytical models and quantitative synthetic models of the dynamic evolution. This absence makes it difficult to justify analysis and synthesis choices and prevents a deductive approach to longitudinal dynamics. Within the framework of a 3-year longitudinal empirical and technological research on appropriation, this article discusses the conditions of production and the usefulness of a quantitative synthetic model of the longitudinal complexity of individual activity, in conjunction with a qualitative analytical method. More than the mobilization of a single, pre-data model, the modelling work involves an active articulation between analytical model-methods (classical in activity analysis) and synthetic model-methods. This linkage offers prospects for improving longitudinal studies centred on human activity and provides useful visual support for design projects. We discuss the contributions and limits of this work with respect to activity analysis in French-language ergonomics and the complexity sciences most often mobilized in human factors.

\section{INDEX}

Mots-clés : modélisation, modèle synthétique, activité individuelle, étude longitudinale, complexité

Keywords : modelling, individual activity, longitudinal study, complexity, synthetic model

\section{AUTEUR}

\section{JULIEN GUIBOURDENCHE}

AKTEN et Université de Genève, 2 chemin de la petite Cerdagne, 69270 Fontaines-sur-Saône julien.guibourdenche@akten.fr 